The Egyptian International Journal of Engineering Sciences \& Technology, Vol 3, No 1 (1999)

\title{
Methods Used For Solving Project Management Problems And Thei Comparisons
}

AbdelRazek AbuolNour, EISayed A. ElSayed

\begin{abstract}
The major results gained from research is entering some suitable adjustment for conventional planning techniques to able as control tool in updating, clearing to many divisions for precedence diagram, PD. $\quad$ Finally a macro model for project planning techniques called project planning family tree is given
\end{abstract}

